\title{
Review: studies with sufficient follow-up do not show a clear benefit for pharmacotherapy in alcohol dependence
}

Garbutt JC, West SL, Carey TS, et al.Pharmacological treatment of alcohol dependence. A review of the evidence.JAMA 1999 Apr14;281:1318-25.

\author{
QUESTION: What is the efficacy of 5 classes of drugs (disulfiram, opioid antagonists \\ [naltrexone and nalmefene], acamprosate, serotonergic agents, and lithium) for treating \\ alcohol dependence?
}

\section{Data sources}

Studies were identified by searching Medline, HealthSTAR, the American Society of Health-System Pharmacists International Pharmaceutical Abstracts, EMBASE/ Excerpta Medica, Alcohol and Alcohol Problems Database, PsycINFO, and the Cochrane Library with terms for drugs of interest and related classes of drugs, alcoholism, alcohol drinking, and study design characteristics. Published abstracts and US Food and Drug Administration documents were also reviewed.

\section{Study selection}

Studies published in English, French, or German from 1966 to December 1997 were selected if they included $\geq 10$ adults who were dependent on alcohol and treated in any setting. Children, infants, and pregnant women were excluded.

\section{Data extraction}

Reviewers assessed the quality of studies by using a quality rating score and extracted data on participants, follow-up, trial duration, number of drinking and non-drinking days, return to drinking, time to first drink, alcohol consumed per unit of time, craving, and relapse.

\section{Main results}

41 studies met the selection criteria. 5 randomised controlled trials $(\mathrm{RCTs})(\mathrm{n}=1207)$ used a double-blind, placebo-controlled design to evaluate oral disulfiram, 200-250 mg/day; only 2 studies reported > $>80 \%$ follow-up. One of these studies reported a beneficial effect for drinking days but no effect for return to drinking or time to first drink; the other study reported no effect for drinking days and alcohol consumed per unit of time. 6 RCTs ( $n=282,5$ placebo-controlled) evaluated disulfiram implants, $800-1000 \mathrm{mg}$; but only 2 studies reported $>80 \%$ follow-up. Both RCTs reported a lack of effect on drinking days, time to first drink, and alcohol consumed per unit of time; 1 RCT reported a lack of effect on return to drinking. 8 RCTs evaluated serotonergic agents in patients with comorbid conditions. 5 double-blind, placebo-controlled RCTs $(\mathrm{n}=227)$ used fluoxetine. Only 2 of these RCTs reported $>80 \%$ follow-up, and neither reported a beneficial effect. 1 RCT evaluated citalopram, 1 evaluated ondansetron, and 1 evaluated buspirone. None reported $>80 \%$ follow-up. In patients with anxiety or mood disorders, only 1 RCT $(\mathrm{n}=51)$ had $>80 \%$ follow-up, and it reported a beneficial effect for fluoxetine on drinking days and alcohol consumed per unit of time but no effect for return to drinking and time to first drink. RCTs that evaluated naltrexone, $50 \mathrm{mg} /$ day (3 placebo-controlled, $\mathrm{n}=271$ ); acamprosate, 1300-2000 $\mathrm{mg}$ /day (9 double-blind, $\mathrm{n}=2170$ ); and lithium (6 RCTs, $\mathrm{n}=823$ ) had $<80 \%$ follow-up.

\section{Conclusions}

Many studies on alcohol dependence do not have sufficient follow-up. Studies with sufficient follow-up do not show a clear benefit for pharmacotherapy in alcohol dependence.

\section{COMMENTARY}

This carefully done systematic review by Garbutt and colleagues summarises the current evidence. The authors concluded that the overall evidence shows that the opiate antagonist naltrexone and acamprosate were "clearly superior to placebo for treatment of alcohol dependence."* Some of the positive experiences from alcohol seem mediated through opiate mechanisms, and naltrexone substantially decreased the rate of relapse to heavy drinking and the frequency of drinking. Acamprosate, with effects on $\gamma$-aminobutyric acid and other neurotransmitters, is approved in Europe and is undergoing several clinical trials in the United States but is not yet released. Well-designed RCTs consistently showed more substantial improvement in nondrinking and drinking days with acamprosate than with placebo.

These medications were always used in conjunction with psychosocial interventions, a practice that should continue. None of the drugs represents a pharmacological "cure" for alcoholism. Many more studies are needed to better define their place in alcoholism treatment, including timing and duration of treatment and appropriate patient populations. Nevertheless, whereas the high rate of patient dropouts is a common issue in alcohol studies, valuable knowledge is steadily being gained about this increasingly important element in alcoholism treatment.

Michael Mayo-Smith, MD, MPH
Veterans Affairs Medical Center
Manchester, New Hampshire, USA

*Editor's note

We did not include information on studies of naltrexone and acamprosate in the abstract because the follow-up for these studies did not meet the Evidence-Based Medicine standard of $\geq 80 \%$. The authors point out, however, that this level of follow-up is difficult to achieve in many studies of treatments for alcoholism and in this patient population; the studies that were included in the review met many other stringent quality criteria. 